\title{
Multi-focus Image Fusion Based on Fuzzy and Wavelet Transform
}

\author{
Jamal Saeedi ${ }^{1}$, Karim Faez ${ }^{1}$, and Saeed Mozaffari ${ }^{2}$ \\ ${ }^{1}$ Electrical Engineering Department, Amirkabir University of Technology, Tehran, Iran \\ \{jamal_saeidi,kfaez\}@aut.ac.ir \\ ${ }^{2}$ Semnan University, Electrical and Computer Department, Semnan, Iran \\ mozaffariasemnan.ac.ir
}

\begin{abstract}
In this paper, we proposed a new method for spatially registered multi-focus images fusion. Image fusion based on wavelet transform is the most commonly fusion method, which fuses the source images information in wavelet domain according to some fusion rules. There are some disadvantages in Discrete Wavelet Transform, such as shift variance and poor directionality. Also, because of the uncertainty about the source images contributions to the fused image, designing a good fusion rule to integrate as much information as possible into the fused image becomes one of the most important problem. In order to solve these problems, we proposed a fusion method based on doubledensity dual-tree discrete wavelet transform, which is approximately shift invariant and has more sub-bands per scale for finer frequency decomposition, and fuzzy inference system for fusing wavelet coefficients. This new method provides improved subjective and objectives results compared to the previous wavelet fusion methods.
\end{abstract}

Keywords: Image fusion, double-density dual-tree discrete wavelet transform, fuzzy classifier, multi-focus.

\section{Introduction}

Image fusion provides a means to integrate multiple images into a composite image, which is more appropriate for the purposes of human visual perception and computerprocessing tasks such as segmentation, feature extraction and target recognition. Important applications of the fusion of images include medical imaging [1], microscopic imaging, remote sensing [2], computer vision, and robotics [3].

Fusion techniques include the simplest method of pixel averaging to more complicated methods such as principal component analysis [4], and multi-resolution fusion [5]. Multi-resolution images fusion is a biologically-inspired method, which fuses images at different spatial resolutions. Similar to the human visual system, this fusion approach operates by decomposing the input images into a resolution pyramid of numerous levels. Each level contains one or more bands representing orientation or detail/approximation information. Following this decomposition, the fusion now takes place between the corresponding coefficients or samples in each band. The fused pyramid is then reconstructed to form the final fused output image. 
Nick Kingsbury has introduced DT-CWT [6], which introduces limited redundancy (4X) and allows the transform providing approximate shift invariance and directionally selective filters while preserving the usual properties of perfect reconstruction and computational efficiency. There are many publications, which used DT-CWT for fusion schemes and showed better subjective and objective results [7]. In this paper we proposed a new algorithm based on double-density dual-tree DWT [8], which is an over complete discrete wavelet transform (DWT) designed to simultaneously possess the properties of the double-density DWT [9], and the dual-tree complex DWT [6].

The three previously important developed fusion methods, which were implemented in wavelet transform domain, are as follows: Maximum selection (MS), which just picks the coefficients in each sub-band with the largest magnitude; Weighted average (WA), which is developed by Burt and Kolczynski [10] and used a normalized correlation between the two images sub-bands over a small local area. The resultant coefficients for reconstruction are calculated from this measure via a weighted average of the two images coefficients; Window based verification (WBV), which is developed by Li et al. [11] and creates a binary decision map to choose between each pair of coefficients using a majority filter.

These fusion rules ignore some useful information and are sensitive to noise. Selective operation made the fused coefficients completely dependent on the coefficients with larger average of local area energy and ignores the other corresponding coefficient. In the weighted average scheme, the weights were computed by a linear function which cannot describe the uncertainty of each source image contributions. Also in coarser level of decomposition, fusion task is much harder and these fusion rules do not work very well. In order to solve these uncertainties and information integration, this paper proposed a new fusion algorithm, which also is based on new wavelet transform and new fusion rule based on fuzzy classifier.

The paper is structured as follows: In Section 2 we describe briefly about the double density dual tree DWT. In section 3 the proposed fuzzy image fusion is explained. Section 4 gives various results and comparisons. Finally, we conclude with a brief summary in section 5 .

\section{Double-Density Dual-Tree DWT}

A double-density dual-tree DWT [8] is proposed by Selesnick in 2004. Important refinements in DD-DT-DWT provide filters that are nearly shift-invariant with vanishing moments, compact support, and a high degree of smoothness.

The 2-D DD-DT-DWT has a total of 32 oriented real wavelets or 16 complex wavelet sub-images per level, while the DT-DWT has 12 oriented real wavelets or 6 complex wavelet sub-bands filters per level. This structure is sometimes described by a parent children- grandchildren genealogy (e.g. parents start at level 3, children at level 2, and grandchildren at level 1). The DD-DT-DWT by comparison with the DTDWT can has wavelets, which are more closely spaced spatially, or wavelet subbands which are more closely spaced with respect to scale. It also has more sub-bands per scale for finer frequency decomposition with increased wavelet regularity for same length filters. However, 2-D DD-DT-DWT requires 10.66X rather than 4X memory increase in DT-DWT. Nevertheless, because of their finer regular sub band coverage, the DD-DT-DWT will be used in this research. 


\section{Proposed Fuzzy Image Fusion}

In this scheme, the fusion output achieved by combination of three inputs obtained with three different fusion rules. In fact the fuzzy system specifies three inputs contribution in the final output. These fusion rules can be explained as follows:

\subsection{Fusion Using Decision Map}

This rule forms the first input of the fuzzy system using a logical matrix, called decision map. In many publications local features is used to generate the decision map, for selecting coefficients between high frequency sub-bands of two images such as mean and standard deviation [12], energy, gradient, fractal dimension, contrast, and standard deviation [2], spatial frequency, visibility, and information entropy [13], for image fusion. Here, we used a combination of two features for generating confident decision map.

The high frequency coefficients reflect the image edge and detail information. According to imaging mechanism of optical system, the bandwidth of system function for images in focus is wider than that for images out of focus. Therefore the pixel values of clear images are larger than that of blurred images.

In order to enhance this information, we used two texture features. The first feature calculates local range and second one calculates local energy of the high-frequency sub-bands. We calculate the two features using:

$$
\begin{gathered}
\text { Range: } F_{1}{ }^{k}(x, y)=\max _{x, y \in W}\left|s b_{k_{i}}^{j}(x, y)\right|-\min _{x, y \in W}\left|s b_{k_{i}}^{j}(x, y)\right| \\
\text { Energy: } F_{2}{ }^{k}(x, y)=\sum_{x, y \in W}\left(s b_{k_{i}}^{j}(x, y)\right)^{2}
\end{gathered}
$$

where $j=1,2 \ldots N-1$, which $N$ is the level of the decomposition, $i=1,2 \ldots 16$, which denote the sixteen sub-bands of high frequency coefficients at each level, $k=1,2$, which is the number of images, and $W$ is the local window.

Also for improving these features a nonlinear averaging filter is used for reducing noise and taking into accounts neighbor dependency. This operation implement as follows:

$$
N F_{i}^{k}(x, y)=\frac{\sum_{a=-A}^{A} \sum_{b=-B}^{B} \mu(a, b) \times F_{i}^{k}(x, y)}{\sum_{a=-A}^{A} \sum_{b=-B}^{B} \mu(a, b)}
$$

where $i=1,2$, which is two texture features, $k=1,2$ is the number of images and $[2 \times A+1,2 \times B+1]$ is the size of local window. Also $\mu(a, b)$ is calculated using:

$$
\mu(a, b)=\exp \left[-\left(\frac{a^{2}+b^{2}}{N W}\right)\right]
$$




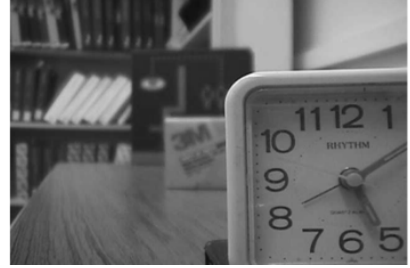

(a)

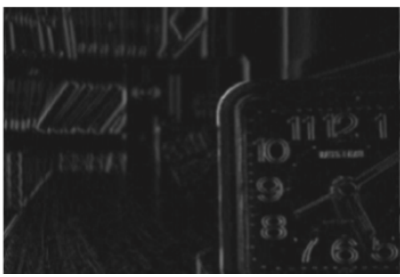

(b)

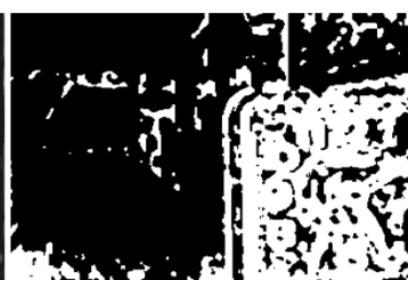

(c)

Fig. 1. (a) Right-focus "Disk" image. (b) 2th Sub-band at first level of decomposition for right focus image. (c) Decision map for 2th sub-band.

where $N W$ is the number of pixel in the local window and $a \in\{-A \ldots A\}, b \in$ $\{-B \ldots B\}$. Having the two features, decision map is calculated using:

$$
d m_{i}^{j}=\left\{\begin{array}{ccc}
1 & \text { if } & N F_{1}^{1}>N F_{1}^{2} \text { and } N F_{2}^{1}>N F_{2}^{2} \\
0 & \text { otherwise }
\end{array}\right.
$$

For example a decision map is obtained for the "Disk" images, which shows in the Figure 1. Finally the first rule output is calculated using:

$$
Y_{1}=d m_{i}^{j} \times s b_{1 i}^{j}+\left(1-d m_{i}^{j}\right) \times s b_{2 i}^{j}
$$

\subsection{Fusion Using Finer Level Decision Map}

Most of the fusion rules for merging wavelet coefficients [10], [11] do not work well in coarser level of decomposition. This is happened because of in the coarser level of decomposition there is not sufficient different between features for generation of desired decision map. Therefore we used an estimation of finer level decision map via down-sampling or interpolation for fusing sub-band of two images in coarser levels. Figure 2 shows estimation of decision map for coarser level. The output of second rule can be defined by following equation:

$$
Y_{2}=d m_{i}^{j-1} \times s b_{1 i}^{j}+\left(1-d m_{i}^{j-1}\right) \times s b_{2 i}^{j}
$$

where $j=2,3 \ldots N-1$, which $N$ is the level of the decomposition, $i=1,2 \ldots 16$, which denote the sixteen sub-bands of high frequency coefficients at each level. For $j=1, \mathrm{Y}_{2}$ obtained via first rule.

Spatial correlation between wavelet coefficients in different levels, which is called inter-scale dependency is the idea behind this fusion rule, which is used in many publication for wavelet based compression and denoising [14].

\subsection{Fusion Using Averaging}

In the smooth region of two images that may be existed in focus or out of focus region in the image we cannot take a good decision for fusion task, because there is not sufficient difference between them for distinguishing in and out of focus regions. Therefore 
we used a simple fusion rule, which is averaging that can remove Gaussian noise. This rule can be defined using:

$$
Y_{3}=\frac{s b_{1 i}^{j}+s b_{2 i}^{j}}{2}
$$

\subsection{Fuzzy Classifier}

We want to design a good fusion rule with combining these three fusion rules to integrate as much information as possible into the fused image. We used a fuzzy classifier for this purpose.

Here we used fuzzy rule-based classifier. The simplest fuzzy rule-based classifier is a fuzzy if-then system, similar to that used in fuzzy control [15]. We labeled output of each fusion rules as a class. This classifier can be constructed by specifying classification rules as linguistic rules:

1. IF $\mathrm{NF}_{1}$ is large $\mathrm{AND} \mathrm{NF}_{2}$ is large THEN $\mathrm{Y}_{1}$ is output.

2. IF $\mathrm{NF}_{1}$ is large $\mathrm{AND} \mathrm{NF}_{2}$ is small THEN $\mathrm{Y}_{2}$ is output.

3. IF $\mathrm{NF}_{1}$ is medium $\mathrm{AND} \mathrm{NF}_{2}$ is large THEN $\mathrm{Y}_{1}$ is output.

4. IF $\mathrm{NF}_{1}$ is medium $\mathrm{AND} \mathrm{NF}_{2}$ is small THEN $\mathrm{Y}_{2}$ is output.

5. IF $\mathrm{NF}_{1}$ is small THEN $\mathrm{Y}_{3}$ is output.

where $N F_{1}=\left|N F_{1}^{1}-N F_{1}^{2}\right|$, and $N F_{2}=\left|N F_{2}^{1}-N F_{2}^{2}\right|$.

Each linguistic value is represented by a membership function. Figure 3 shows triangular membership functions for $N F_{1}$, which is normalized and $T_{1}$ is a constant value. For the pair of values $\left(N F_{1}, N F_{2}\right)$, the degree of satisfaction of the antecedent part of the rule determines the firing strength of the rule. The firing strengths of these rules are calculated as:
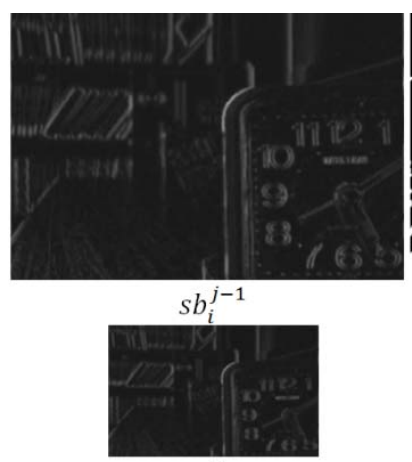

$s b_{i}^{j}$
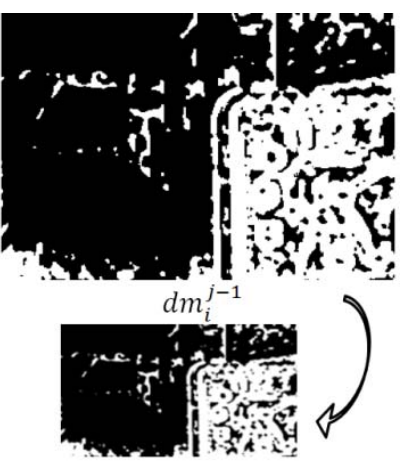

$d m^{*}$

Fig. 2. Estimation of decision map $\left(\boldsymbol{d m} \boldsymbol{m}^{*}\right)$ for coarser level 


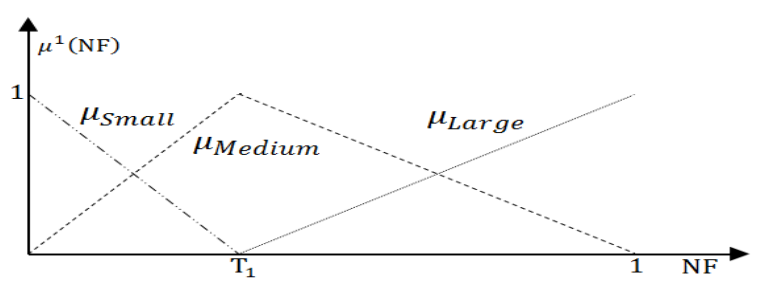

Fig. 3. Fuzzy membership function for the linguistic terms of $\boldsymbol{N F}_{\mathbf{1}}$

$$
\begin{gathered}
\tau_{1}=\mu_{\text {large }}^{1}\left(N F_{1}\right) \times \mu_{\text {large }}^{2}\left(N F_{2}\right), \tau_{2}=\mu_{\text {large }}^{1}\left(N F_{1}\right) \times \mu_{\text {small }}^{2}\left(N F_{2}\right) \\
\tau_{3}=\mu_{\text {medium }}^{1}\left(N F_{1}\right) \times \mu_{\text {large }}^{2}\left(N F_{2}\right), \tau_{4}=\mu_{\text {medium }}^{1}\left(N F_{1}\right) \times \mu_{\text {small }}^{2}\left(N F_{2}\right) \tau_{5}=\mu_{\text {small }}^{1}\left(N F_{1}\right)
\end{gathered}
$$

The AND operation is typically implemented as minimum but any other t-norm may be used. We have chosen algebraic product for the AND operation.

The rules "vote" for the class of the consequent part. The weight of this vote is $\tau_{i}$. To find the output of the classifier, the votes of all rules are aggregated. Among the variety of methods that can be applied for this aggregation, we considered the maximum aggregation method. Let $\mathrm{k}$ is the class labels, $\mathrm{j}$ denote number of rules and $i \rightarrow k$ denote that rule $\mathrm{i}$ votes for $\mathrm{Y}_{\mathrm{k}}$. Then:

$$
\text { If } \tau_{i}=\max _{j=1 \ldots 5} \tau_{j} \quad \text { AND } \quad i \rightarrow k \quad \text { THEN Class is } Y_{k}
$$

For building fuzzy membership function $T_{1}$ must be defined. We obtained $0.1 \leq T_{1} \leq$ 0.2 using test images and try and error. Finally the new sub-band for generating output image is obtained using:

$$
s b_{-} n e w_{i}^{j}=Y_{k}
$$

where $j=1,2 \ldots N-1$, which $\mathrm{N}$ is the level of the decomposition, $i=1,2 \ldots 16$, which denote the sixteen sub-bands of high frequency coefficients at each level.

Also fusion rule for low-frequency sub-bands is defined by:

$$
s b_{-} n e w_{i}^{N}=\frac{s b_{1 i}^{N}+s b_{2 i}^{N}}{2}
$$

where $i=1,2$, which is low frequency sub-bands in the last level. After merging the wavelet coefficients, the final fusion result is obtained by inverse wavelet transform.

\section{Experimental Results}

The images used in these experiments are selected from multi-focus datasets; publicly available at the Image fusion web site [18] (Figures 4). To compare our image fusion method, the image fusions based on the DWT [5], shift invariant DWT (SIDWT) [16], and DT-CWT decompositions [8] are also implemented.

To evaluate our comparisons objectively, the same fusion rules (MS, WA [10], WBV [11], and our proposed method) are used in the DWT, SIDWT, DT-CWT and DD-DT-DWT schemes. The image PSNR and Petrovic index [17], used to evaluate 


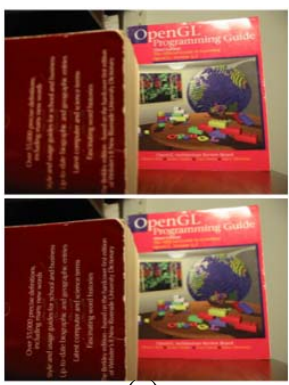

(a)

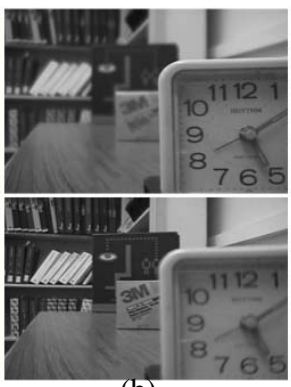

(b)

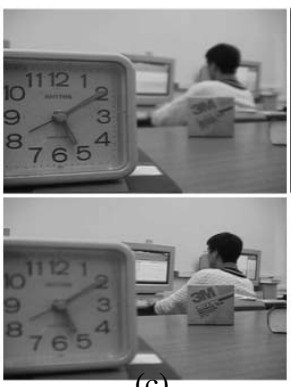

(c)

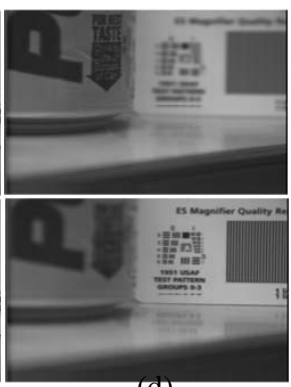

(d)

Fig. 4. Test images used in the experiments. (a)-(d) Book, Disk, Lab and Pepsi, respectively.

the fused image. It should be mentioned that for image fusion experiment, a groundtruth image was used by cutting and pasting method. Subjective results show better visual effect without any artifact compared to other fusion schemes. Notice to the artifacts around the head in the Figure 5 (a)-(c) images. Also objective results in the Tables 1 obviously indicate that our fusion scheme is better than others.

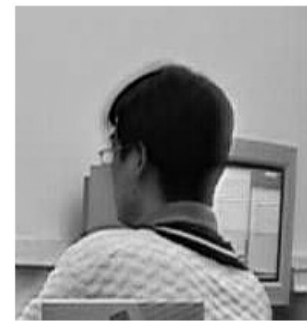

(a)

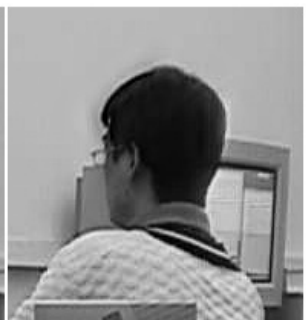

(b)

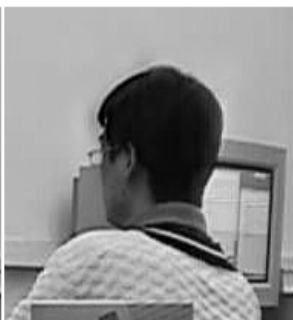

(c)

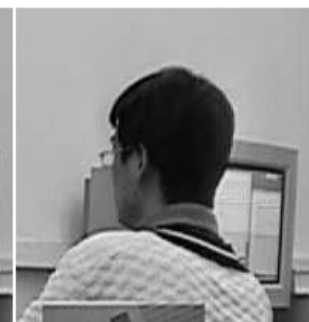

(d)

Fig. 5. Subjective fusion results, Fusion result of a part of "Lab" image using DD-DT-CWT and (c) MS (d) WA (e) WBV (f) proposed method

Table 1. objective image fusion results

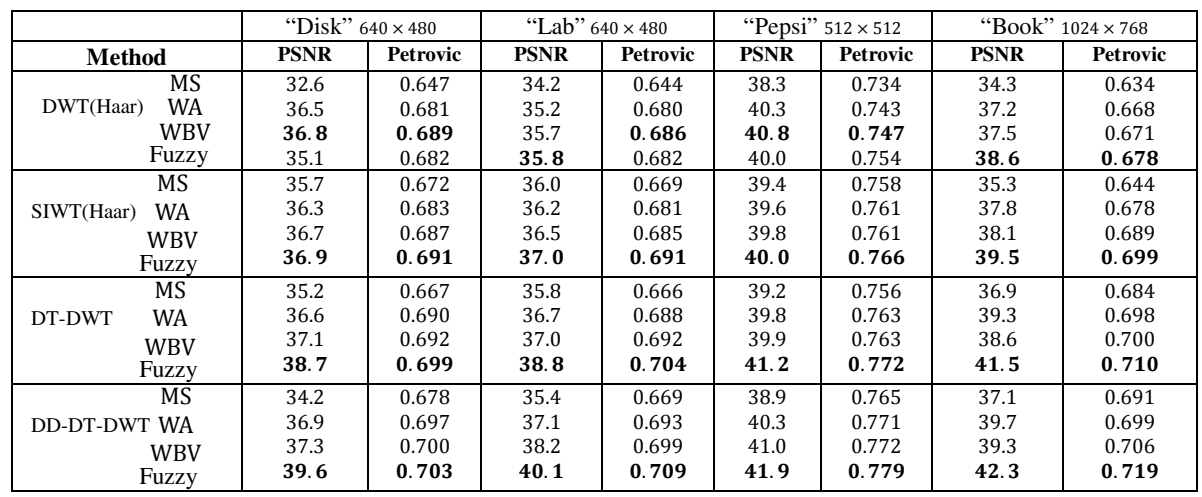




\section{Conclusion}

In this paper, we have presented a new multi-focus image fusion method using double-density dual-tree DWT and fuzzy classifier. This new method used DD-DTDWT for finer frequency decomposition and shift invariant property compared to other wavelet decomposition and fuzzy classifier for fusing sub-bands of two images, because of overcoming uncertainties in other fusion algorithm mentioned before. The experimental results demonstrated that the proposed method outperforms the standard wavelet fusion methods in the fusion of multi-focus images.

\section{References}

1. Garg, S., Kiran, U., Mohan, K., Tiwary, R.: Multilevel Medical Image Fusion using Segmented Image by Level Set Evolution with Region Competition. In: 27th Annual International Conference of the Engineering in Medicine and Biology Society, January 17-18, pp. 7680-7683 (2006)

2. Yang, X.-H., Jing, Z.-L., Liu, G., Hua, L.Z.: Fusion of multi-spectral and panchromatic images using fuzzy rule. Communications in Nonlinear Science and Numerical Simulation 12, 1334-1350 (2007)

3. Kam, M., Zhu, X., Kalata, P.: Sensor fusion for mobile robot navigation. Proceedings of the IEEE 85, 108-119 (1997)

4. Kumar, S., Senthil, M.S.: PCA-based image fusion. In: Proceedings of the SPIE, vol. 6233, p. 62331T (2006)

5. Ke, R.Z., Li, Y.-J.: An Image Fusion Algorithm Using Wavelet Transform. Acta Electronica Sinica 32(5), 750-775 (2004)

6. Kingsbury, N.: A Dual-Tree Complex Wavelet Transform with Improved Orthogonality and Symmetry Properties. In: ICIP, vol. 2, pp. 375-378 (2000)

7. Wei, S., Ke, W.: A Multi-Focus Image Fusion Algorithm with DT-CWT. In: International Conference on Computational Intelligence and Security, pp. 147-151 (2007)

8. Selesnick, I.W.: The Double-Density Dual-Tree DWT. IEEE Trans. on Signal Processing 52(5), 1304-1314 (2004)

9. Petrosian, Meyer, F.G.: The double density DWT. In: Wavelets in Signal and Image Analysis: From Theory to Practice, Kluwer, Boston (2001)

10. Burt, P.J., Kolczynski, R.J.: Enhanced image capture through fusion. In: Proceedings of the 4th International Conference on Computer Vision, pp. 173-182 (1993)

11. Li, H., Manjunath, B.S., Mitra, S.K.: Multi-sensor image fusion using the wavelet transform. Graphical Models and Image Processing 57(3), 235-245 (1995)

12. Arivazhagan, S., Ganesan, L., Subash Kumar, T.G.: A modified statistical approach for image fusion using wavelet transform. Springer, London (2008)

13. Li, S., Kwok, J.T.: Multi-focus image fusion using artificial neural networks. Pattern Recognition Letters 23, 985-997 (2002)

14. Sendur, L., Selesnick, I.W.: Bivariate Shrinkage Functions for Wavelet-Based Denoising Exploiting Interscale Dependency. IEEE Transactions on Signal Processing 50(11), 2744 2755 (2002)

15. Kuncheva, L.I.: Fuzzy Classifier Design. Springer, Heidelberg (2000)

16. Rockinger, O.: Image Sequence Fusion Using a Shift Invariant Wavelet Transform. In: ICIP, pp. 288-291 (1997)

17. Petrović, V., Xydeas, C.: Evaluation of image fusion performance with visible differences. In: Pajdla, T., Matas, J(G.) (eds.) ECCV 2004. LNCS, vol. 3023, pp. 380-391. Springer, Heidelberg (2004)

18. http://imagefusion.org 